\title{
Deontological morality can be experimentally enhanced by increasing disgust: A transcranial direct current stimulation study
}

\author{
Cristina Ottaviani $^{\mathrm{a}, \mathrm{b}}$, Francesco Mancinic ${ }^{\mathrm{c}, \mathrm{d}, *}$, Samantha Provenzano ${ }^{\mathrm{b}}$, Alberto Collazzoni ${ }^{\mathrm{c}}$, \\ Francesca D'Olimpio ${ }^{\mathrm{e}}$
}

a Department of Psychology, Sapienza University of Rome, Rome, Italy

b Neuroimaging Laboratory, IRCCS Santa Lucia Foundation, Rome, Italy

c Scuola di Psicoterapia Cognitiva S.r.l., Rome, Italy

d Guglielmo Marconi University, Rome, Italy

e Department of Psychology, University of Campania "Luigi Vanvitelli", Caserta, Italy

A R T I C L E I N F O

\section{Keywords:}

Transcranial current direct stimulation

Obsessive-compulsive disorder

Heart rate variability

Disgust

Insula

Morality

\begin{abstract}
A B S T R A C T
Previous studies empirically support the existence of a distinctive association between deontological (but not altruistic) guilt and both disgust and obsessive-compulsive (OC) symptoms. Given that the neural substrate underlying deontological guilt comprises brain regions strictly implicated in the emotion of disgust (i.e. the insula), the present study aimed to test the hypothesis that indirect stimulation of the insula via transcranial direct current stimulation (tDCS) would enhance disgust and morality in the deontological domain. A randomized, sham-controlled, within-subject design was used. Thirty-seven healthy individuals (25 women) underwent 15-min anodal and sham tDCS over T3 in two different days, while their heart rate (HR) was recorded to derive measures of parasympathetic nervous system activity (HR variability; HRV). After the first 10-min of sham or active tDCS stimulation, participants were asked to 1) complete a series of 6-item words that could be completed with either a disgust-related word (cleaning/dirtiness) or neutral alternatives; 2) rate how much a series of vignettes, each depicting a behavior that violated a specific moral foundation, were morally wrong. Levels of trait anxiety, depression, disgust sensitivity, scrupulosity, and altruism as well as pre- and post- stimulation momentary emotional states were assessed. Compared to the sham condition, after active stimulation of T3 a) HRV significantly increased and participants b) completed more words in terms of cleaning/dirtiness and c) reported greater subjective levels of disgust, all suggesting the elicitation of the emotion of disgust. Although the results are only marginally significant, they point to the absence of difference between the two experimental conditions for moral vignettes in the altruistic domain (i.e., animal care, emotional and physical human care), but not in the deontological domain (i.e., authority, fairness, liberty, and sacrality), where vignettes were judged as more morally wrong in the active compared to the sham condition. Moreover, scores on the OCI-R correlated with how much vignettes were evaluated as morally wrong in the deontological domain only. Results preliminarily support the association between disgust and morality in the deontological domain, with important implications for OC disorder (OCD). Future studies should explore the possibility of decreasing both disgust and morality in patients with OCD by the use of non-invasive brain stimulation techniques.
\end{abstract}

\section{Introduction}

In all religions, sin dirties the conscience and washing the body cleanses the conscience. Empirical studies support the idea that the association between morality and disgust goes beyond mere metaphor (Lee and Schwarz, 2011; Schnall et al., 2008a, 2008b). In Zhong and Liljenquist's seminal study, the recall of unethical acts induced the need to cleanse oneself (the so called "Lady Macbeth effect", as demonstrated "through an increased mental accessibility of cleansing-related concepts, a greater desire for cleansing products, and a greater likelihood of taking antiseptic wipes" (Zhong and Liljenquist, 2006, p. 1451)). Moreover, physical cleansing helps reducing threats to one's moral selfimage (Zhong and Liljenquist, 2006).

Physical and moral disgust share some features such as the fear of contamination. Mental contamination occurs in the absence of direct contact with a contaminant, and is seen for example when victims of

\footnotetext{
* Correspondence to: Scuola di Psicoterapia Cognitiva S.r.l., Viale Castro Pretorio, 116, 00185 Rome, Italy.

E-mail address: mancini@apc.it (F. Mancini).
} 
immoral acts, such as rape, develop strong feelings of contamination, and associated compulsive washing (Herba and Rachman, 2007; Rachman, 2004). Mental contamination is a characteristic of obsessivecompulsive disorder (OCD), it can be worsened by the addition of elements of betrayal (Rachman et al., 2012), and seems to have a moral quality that, in our view, is deontological in nature. In OCD, disgust serves to protect oneself from mental contamination, in the sense of safeguarding the self from deontological degradation.

The Lady Macbeth effect has not always been replicated (Earp et al., 2014). In our view, such inconsistencies may be due to the fact that studies usually ask participants to recall an ethical or unethical deed, without considering an important distinction within the domain of guilt; i.e. the distinction between altruistic and deontological guilt (Mancini and Gangemi, 2017). The first appears when one appraises one's own conduct as not being altruistic (e.g., not having shared a victim's destiny such as in survivor guilt, or not having been close to her/him under difficult circumstances like a health disease), and the second arises out of the assumption of having violated one's own moral rules. In altruistic guilt, there is always a victim, but there may not have been any violation of moral rules. In deontological guilt, on the contrary, there may be no victim (e.g., incest between consenting siblings), or one could feel deontological guilt even when acting for the good of a victim as in the case of euthanasia, where -to reduce the victim's suffering- the moral norm of "Not Play God" has to be violated. Thus in deontological guilt, the assumption of having violated a moral rule is necessary and sufficient.

In the last decade, empirical evidence supporting the distinction between the two types of guilt has been accumulated. For example, the work of D'Olimpio and Mancini (2014) showed that the induction of deontological guilt triggered more obsessive compulsive-like washing behaviors compared to the induction of altruistic guilt. This effect was replicated and extended by Ottaviani et al. (2018), who also found that compared to altruistic guilt, inducing deontological guilt enhanced vagally-mediated heart rate variability (HRV). HRV is a proxy for parasympathetic activation (Rohrmann and Hopp, 2008), and has been used as a useful indicator to examine the relative contribution of disgust and fear (Cisler et al., 2009; Davey, 2011). In fact, the topography of autonomic nervous system response is distinct for general stress/fear and disgust. The former being characterized by phasic HRV suppression, representing the withdrawal of cardiac vagal control and the activation of the defensive systems (e.g., Park et al., 2014), and the latter being characterized by HRV increases, due to baroreceptor activation and parasympathetic dominance (Ekman et al., 1983; Schlegel et al., 2001). During disgust, HRV increases mainly because disgust-related functions such as monitoring of tastes, rejection of inedible foods, nausea and vomiting are achieved by activation of the vagus nerve parasympathetic drive to the heart via brainstem nuclei (Babic and Browning, 2014).

Importantly, a neuroimaging study by Basile et al. (2011) found that the induction of deontological guilt increased activation of brain areas implicated in the emotion of disgust, such as the insula (Pujol et al., 2018), whereas altruistic guilt elicited activation in the theory of mind network, particularly in medial prefrontal brain areas. Taken together, these studies suggest that the Lady Macbeth effect may be more strictly connected with deontological rather than altruistic guilt. Indeed, in the two emblematic cases of Pontius Pilatus and the baptism, the act of washing is related to deontological guilt.

Other studies based on the affect theory, demonstrated that the severity of moral judgment can be amplified by disgust (amplification hypothesis). For instance, Tobia (2015) reported that the sensation of dirtiness elicits a stronger tendency to judge oneself more negatively from a moral point of view. Similarly, Rozin and colleagues (Rozin et al., 1999) found that people tend to associate disgust with purity violations that have greater conceptual relation to deontological rather than altruistic guilt. A meta-analysis of studies in which incidental disgust was manipulated prior to or concurrent with a moral judgment task, found only a small effect size, which disappeared after controlling for publication bias (Landy and Goodwin, 2015). Unexpectedly, the authors found that the amplification effect is not restricted to moral transgressions in the purity domain. This result could be considered contrary the hypothesis of a distinctive bidirectional effect between disgust and the deontological domain. However, in our view some of the studies described in the meta-analytic work by Landy and Goodwin (2015) used paradigms that do not take into account the altruistic option but instead oppose deontological and consequentialist choices (i.e., producing more overall good than any alternative action). Moreover, the deontological choice is often spurious and confounded with altruistic motivations, like in the so-called "footbridge dilemma." In this dilemma, you can save five people from a runaway trolley by pushing a fat man off the bridge, on to the track below. This scenario does not distinguish between altruistic and deontological guilt, because one can opt for the deontological choice of omission for two different motivations: the so-called "Do not play God" (deontological motivation), or because of empathic feelings toward the fat man (altruistic motivation). Indeed, as suggested by authors of the meta-analysis, it is necessary to develop procedures that induce core disgust in ways that do not transgress social norms, as some of these inductions actually imply moral disapproval or small harm to participants (Landy and Goodwin, 2015).

The current study aims to provide further insight into the relationship between morality and disgust. Our overall hypothesis is that deontological -but not altruistic- morality is distinctively associated with the emotion of disgust, and this may, at least partially, explain existing contradictory findings on the Lady Macbeth effect.

Drawing on preexisting neuroimaging evidence on the association between deontological guilt and brain regions associated with disgust (e.g., the insula), we used transcranial direct current stimulation (tDCS) to indirectly stimulate the insula. A series of previous studies show that with this methodology it is possible to stimulate the areas located just below the temporal cortex, comprising the insula by the use of tDCS over T3 (Montenegro et al., 2011; Okano et al., 2015; Petrocchi et al., 2017; Piccirillo et al., 2016). If deontological -but not altruistic- guilt is strictly connected with the emotion of disgust, and they share part of the same neural network, i.e. the insula, then our prediction would be that anodal stimulation over T3 (versus sham) elicits the emotion of disgust in non-pathological individuals via indirect stimulation of the insula. To obtain a comprehensive assessment of disgust, we combined three different types of measures: subjective self-report, physiological, and implicit measures. This is in line with the suggestion of Landy and Goodwin (2015), because the definition of core disgust does not imply the violation of social norms, and it offers different measures of the construct of disgust.

The use of implicit measures is based on the recognition that emotional cognitive processes can occur implicitly (Winkielman and Berridge, 2004). The implicit measure used in the present study is the word fragment completion task, which assesses the extent to which affective information is accessible from memory (e.g., Anderson et al., 2003; Denny and Hunt, 1992).

Our second predicted result is that anodal stimulation over T3 (versus sham) would enhance moral judgment in the deontological but not in the altruistic domain. To assess moral judgment in these two domains, we used the Moral Foundations Vignettes developed by Clifford et al. (2015), which are based on the domains of the Moral Foundation Theory, an influential scientific account of morality designed to explain the variety and universality of moral judgments (Graham et al., 2011). The theory incorporates psychological, developmental, and evolutionary perspectives and proposes that morality is built upon five innate "foundations," each of which selected during human evolution and, subsequently, tuned-up by learning during development (see the Moral Task section for a detailed description of the domains).

It is known that the association between morality (and particularly 
deontological morality) and disgust is enhanced in OCD. In particular, Reuven et al. (2014) found a higher Lady Macbeth effect in patients with OCD compared to healthy controls, showing that a higher sensitivity to guilt accounts for washing symptoms in these patients. Moreover, Kang et al. (2016) examined which of the domains represented in Moral Foundations Theory clustered most strongly with obsessivecompulsive symptoms and found that purity/sanctity is the most relevant moral domain. Several studies using fMRI suggest that tasks aimed at eliciting OC symptoms in these patients activate the same areas that are involved when non-pathological individuals are experiencing deontological guilt, in particular, the anterior cingulate cortex and the insulae (Basile et al., 2011; Mataix-Cols et al., 2005; Rauch et al., 1998). Lastly, results from Ottaviani et al. (2013) suggest that immorality relies on the same biological root of physical disgust only in subjects with obsessive-compulsive tendencies. Given these premises, our last hypothesized result is that the increase in levels of disgust and deontological morality after stimulation of the insular region would be stronger in participants with higher obsessive-compulsive dispositional tendencies.

\section{Methods and materials}

\subsection{Participants}

Participants were recruited from public advertisements in social networks; the final sample was mostly composed by graduate and postgraduate students of several universities in Rome.

Forty-one individuals agreed to participate in the study and completed a pre-screening online questionnaires. Exclusionary criteria were: age younger than 20 years and self-reported prior history of head injury, major medical neurological or psychiatric disorder, cognitive impairment, history of substance or alcohol abuse or dependence, diagnosis of heart disease, and pregnancy.

After excluding 4 participants because 3 of them reported a psychiatric diagnosis, and one decided to drop out from the study after the first session of stimulation, the final sample was composed of 37 healthy individuals ( 25 women), mean age $=26.78$ (5.04) years. All the participants were Caucasian. All the subjects provided written informed consent and the study has been carried out in accordance with the Code of Ethics of the World Medical Association (Declaration of Helsinki) for experiments involving humans. The study was approved by the Bioethical Committee of S. Lucia Foundation, Rome, Italy. Participants were compensated for their time (30 Euros).

\subsection{Procedure}

Participants were screened by a series of online questionnaires. Eligible participants were asked to avoid drinking coffee and smoking cigarettes for at least $2 \mathrm{~h}$ prior to the sessions. A randomized, shamcontrolled, within-subjects design was used. Each participant underwent anodal and sham tDCS on two different days with a minimum interval between sessions ( $48 \mathrm{~h}$ ). During the entire experimental protocol, participants sat in front of a 15-in. color monitor, at a distance of about $60 \mathrm{~cm}$. First, the HR device was attached using a standard electrode configuration; HR was recorded throughout the entire protocol. Then, participants underwent a 3-min Vanilla baseline assessment during which they were asked to read a neutral magazine (Jennings et al., 1992). At baseline and at the end of the experimental protocol, participants rated their momentary affect on a series of 5-point Likert scales. Participants then underwent a 15-min tDCS sham or active stimulation during which they were asked to rest for the first $10 \mathrm{~min}$, and to complete two subsequent computerized tasks (i.e., moral task and word-stem completion task) for the remaining time ( $5 \mathrm{~min}$ ).

\section{3. $t D C S$}

An anodal or sham tDCS was applied in a counterbalanced random order over T3 (2 mA during $15 \mathrm{~min}$ ), using the BrainSTIM device (EMS s.r.l., Italy). A cathodal tDCS condition was not included for the reason that, whereas it is well established that anodal tDCS increases cortical excitability, the effects of cathodal tDCS are still a matter of debate (Monte-Silva et al., 2010).

The electric current was applied using a pair of sponges soaked in saline solution. The anodal and cathodal electrodes $\left(35 \mathrm{~cm}^{2}\right)$ were connected to a constant current stimulation device with three power batteries $(9 \mathrm{~V})$ presenting a maximal output of $10 \mathrm{~mA}$. For the anodic stimulation targeting left insular cortex, the anode was placed over T3 area according to the international EEG 10-20 system. The cathode was placed over the supraorbital contralateral area (Fp2) and fixed by elastic bands. The electrodes were placed in the same position of the anodal stimulation to perform the sham condition; however, the stimulator was turned off after $30 \mathrm{~s}$. Thus, the subjects reported to feel a tingling or itching sensation coming from the initial electrical stimulation, but they did not receive any further current. This procedure allowed the subjects to remain "blind" in respect to the type of polarity stimulation received during the test and to assure a sham control effect (Gandiga et al., 2006).

\subsection{Computerized tasks}

\subsubsection{Moral task}

E-Prime 2.0 software (Psychology Software Tools) was used for stimulus presentation and response collection. After the first 10-min of stimulation, sentences that described immoral situations were presented in random order on the computer screen. The vignettes used in the present study were adapted from a previously validated large set of moral foundations vignettes (Clifford et al., 2015). These foundations concern dislike for the suffering of others (care/harm), proportional fairness (fairness/cheating), group loyalty (loyalty/betrayal), deference to authority and tradition (authority/subversion), concerns with purity and contamination (sanctity/degradation), and concerns about domination and coercion (liberty/oppression) (Haidt, 2013; Iyer et al., 2012).

The adaptation consisted of two steps: 1) We have decided to exclude the loyalty domain due to the strong cultural dissimilarity between the US and Italy with regard to this concept; 2) We have replaced sacrality items because they were all related to the body, thus leading a bias toward disgust. Sacrality items were created ad-hoc based on the seven deadly sins (e.g., greed, lust).

Sixty-two participants took part in a preliminary study conducted to validate the set in an Italian sample. A link to an online questionnaire was sent via institutional mailing lists and social media. Following the methodology of Clifford et al. (2015), participants were asked to evaluate how much they judged each of the 92 vignettes as morally wrong on a Likert scale from $1=$ not at all to $5=$ very much.

Following this procedure, the 42 vignettes that received the highest scores were selected, 21 of them presented during each tDCS session. Within each group of 21 vignettes, there were 3 vignettes for each specific moral foundation that had been violated: physical animal care ( $\mathrm{n}=3$; e.g., "You see a boy setting a series of traps to kill stray cats in his neighborhood"), emotional care ( $n=3$; e.g., "You see a man snickering as he passes by a cancer patient with a bald head"), physical human care ( $\mathrm{n}=3$; e.g., "You see a girl throwing her hot coffee on a woman who is dating her ex-boyfriend"), authority ( $=3$; e.g., "You see a girl ignoring her father's orders by taking the car after her curfew"), fairness ( $n=3$; e.g., "You see a politician using federal tax dollars to build an extension on his home"), liberty ( $=3$; e.g., "You see a man blocking his wife from leaving home or interacting with others"), and sacrality ( $n=3$; "You see a homosexual in a gay bar offering sex to anyone who buys him a drink"). The first three categories 
(animal, emotional, and physical human care) are attributable to the altruistic domain; whereas authority, fairness, liberty and sacrality belong to the deontological domain. Each vignette depicts a behavior violating a particular moral foundation and not others. The vignettes presented in the two experimental conditions (active vs sham tDCS) were matched within-domain based on the score that they obtained during the preliminary study (e.g., a vignette belonging to the authority domain in the active tDCS condition was matched with a vignette belonging to the same domain and having received the same average score). The vignettes were also controlled on many dimensions including syntactic structure and complexity.

After the first $10 \mathrm{~min}$ of tDCS stimulation (active or sham), the following instructions appeared on the screen: "Please rate how much, in your opinion, each of the following behaviors is morally wrong. To answer, please press the number on the computer keyboard that corresponds to the level of immorality from $1=$ not at all to $5=$ very much". Each sentence appeared in the top of the computer screen followed by the rating scale to remind the possible answers, and they remained visible until a response was given.

The score in each category (e.g., physical human care) was computed as the sum of the scores obtained on three items belonging to that specific category (ranging from 3 to 15). Then, given that the altruistic domain was composed of three categories, whereas the deontological domain was composed of four categories, the total score for "how much is it morally wrong" for each of these two domains was calculated as the average of the scores obtained in the categories belonging to that domain (e.g. for the altruistic domain: sum of the scores obtained on animal care, emotional care, and physical human care divided by 3 ; the total score ranges from 3 to 15 for each domain).

\subsubsection{Word-fragment completion task}

Participants were next asked to complete a 6-item word completion task during which an incomplete word appeared in the center of screen and remained visible until a response was given. This task has been used in previous studies on mental accessibility (e.g., Schimel et al., 2003; Zhong and Liljenquist, 2006).

For each of the 6 words, presented in random order, respondents had to write 3 letters in the consecutive white spaces. Each word could be completed with either a disgust-related word (cleaning/dirtiness) or at least a non-disgust alternative. Examples of stimuli are: S - - - NE (sapone: soap OR salone: salon); DO - - -A (doccia: shower OR doppia: double). Six different words were randomly selected for each session from a total number of 12 words. To avoid conscious deliberation that might contaminate the implicit processing style of the measure, the test instructions encourage respondents to answer each item as quickly as they can and skip any that do not readily come to mind. To account for potential contamination due to cognitive ability, the ratio of target words the participants endorses as disgust-related to that of the total words endorsed was used in the analysis (Johnson and Steinman, 2009).

\subsection{Measures}

\subsubsection{Questionnaires}

Participants completed a set of questionnaires accessing socio-demographic (age and level of education) and lifestyle (nicotine, alcohol, caffeine consumption, medication, and physical activity) information as well as psychological trait and symptoms (e.g., anxiety, disgust sensitivity), and their momentary emotional state.

The trait version of the State-Trait Anxiety Inventory (STAI; Spielberger et al., 1970; Italian translation by Pedrabissi and Santinello, 1989 ) is a 20 -item scale that evaluates relatively stable aspects of "anxiety proneness", including general states of calmness, confidence, and security (e.g., "I am a steady person"). Cronbach's alpha in the present study was 0.92 .

The Center for Epidemiologic Studies Depression Scale (CES-D,
Radloff, 1977; Italian validation by Fava, 1983) is a 20-item self-report scale that assesses the frequency of occurrence of symptoms of depression during the previous week (e.g., "I felt that everything I did was an effort"). Total score ranges from 0 to 60 . Standard cut-offs are 16 for mild depression and 23 for clinical depression. Cronbach's alpha in the present study was 0.73 .

To assess the disgust sensitivity and the obsessive compulsive tendencies, the following tests were used: The Obsessive-Compulsive Inventory-Revised (OCI-R; Foa et al., 2002) as a measure of the presence and severity of obsessions and compulsions, consists of 18 items rated on a 5 -point scale $(0=$ not at all to $4=$ very much) according to the degree of disturbance caused by a behavior (e.g., "I check things more often than necessary"). Cronbach's alpha in the present study was 0.85 .

The Disgust Scale-Revised (DS-R; Olatunji et al., 2007a, 2007b), as a measure of individual differences in sensitivity to disgust, is a 27-items scale composed by two parts: DS-R(a) consists in 14 items rated on a 5point scale $(0=$ totally disagree to $4=$ totally agree) according to the degree of agreement to a sentence (e.g., "I never let any part of my body touch the toilet seat in public restrooms") and DS-R(b) consist in 13 items rated on a 5 -point scale $(0=$ not at all disgusting to $4=$ extremely disgusting) according to the degree of repugnance caused by a situation (e.g., "While you are walking through a tunnel under a railroad track, you smell urine"). Cronbach's alpha in the present study was 0.81 .

The Pennsylvania Inventory of Scrupulosity-Revised (PIOS-R; Olatunji et al., 2007a, 2007b) is a 15 item self-report questionnaire designed to assess the degree of scrupulosity-related symptomatology on a 5 -point scale ( $0=$ never to $4=$ constantly) according to the frequency of subjective experiences (e.g., "I worry that I might have dishonest thoughts"). Cronbach's alpha in the present study was 0.92 .

The Self-Report Altruism Scale (SRAS; Rushton et al., 1981) is a 20item self-report scale asking to rate the frequency with which one has engaged in a series of altruistic behaviors (e.g., "I have donated blood") on a 5 -point scale $(0=$ never to $4=$ very often). Cronbach's alpha in the present study was 0.85 .

The MFQ is designed to assess individual moral system on five moral foundations: Care/Harm, Fairness/Cheating, Loyalty/Betrayal, Authority/Subversion, and Purity/Degradation (Graham et al., 2011). This questionnaire is composed of 2 parts: The first part asks participants to rate, on a 6-point scale, the relevance of various considerations to whether an act is right or wrong (e.g. "Whether someone suffered emotionally"). The second part asks participants to rate, on a 6-point scale, their agreement to various statements (e.g. "Chastity is an important and valuable virtue"). Each part is comprised of 16 items, 3 items corresponding to each of the foundations, and 1 catch item. Cronbach's alpha in the present study was 0.92 .

\subsubsection{Affect rating}

At the beginning and at the end of the experimental protocol, participants were asked to rate their current levels of happiness, shame, fear, sadness, disgust, anger, pity, altruistic guilt, and deontological guilt on a 5 -point Likert scale from $1=$ not at all to $5=$ very much (adapted from D'Olimpio and Mancini, 2014). Given the within-subject design and therefore the need to perform the two tDCS sessions in different days, a change score was computed for each session subtracting the baseline for that specific session from the affect rating after stimulation or sham tDCS (e.g., $\Delta$ happiness $_{\text {stim }}=$ happiness $_{\text {post-stim }}-$

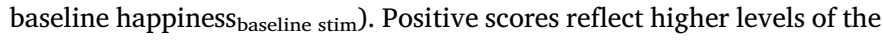
perceived emotional state from baseline to post-tDCS.

\subsection{Physiological data}

HR was recorded as beat-to-beat intervals in ms with the Bodyguard 2 (Firstbeat) device. HRV was assessed by computing the root mean square of successive beat-to-beat interval differences (RMSSD), which 
reflects vagal regulation of HR (Task Force of the European Society of Cardiology, 1996). Outlier and artifact detection, as well as HRV analyses were performed using Kubios HRV software (Tarvainen et al., 2014). From now on, we will use HRV to refer to RMSSD. Given the within-subject design and therefore the need to perform the two tDCS sessions in different days, a change score was computed for each session subtracting the baseline for that specific session from the HRV values during stimulation or sham tDCS (e.g., $\Delta \mathrm{HRV}_{\text {stim }}=\mathrm{HRV}_{\text {during stim }}$ $\mathrm{HRV}_{\text {baseline stim }}$ ). Positive scores reflect and increase in HRV from baseline to post-tDCS.

\subsection{Data analysis}

The analyses were carried out using SPSS Version 21. Differences at $p \leq .05$ are regarded as significant. Data were first checked for outliers, normality of variables, and for violations of statistical assumptions of General Linear Models (GLMs).

First, given that sex differences have been previously acknowledged in moral judgments (e.g., Fumagalli et al., 2010), heart rate variability (Koenig and Thayer, 2016), and neural responses to disgust (Caseras et al., 2007), differences between men and women in the main variables of the study were examined by $t$-test.

Second, to examine the effect of active vs sham tDCS on the elicitation of disgust, a series of univariate analyses of covariance (ANCOVA) with stimulation type as within-subject factor and DS-R score as covariate were performed on the following variables:

a) the ratio of disgust-related words over total recognized words to test for the accessibility of disgust-related information from memory (implicit measure of disgust);

b) $\Delta$ affect rating for each of the examined items (explicit measure of disgust);

c) $\Delta$ HRV (physiological measure of disgust).

To study whether active tDCS enhances moral judgment in the deontological but not in the altruistic domain, we have first obtained two scores based on 1) the average of scores attributed to altruistic vignettes (i.e., animal, emotional, and physical human care), and 2) the average of scores attributed to vignettes in the moral domain (i.e., authority, fairness, liberty, and sacrality). Then, a $2 \times 2$ repeated measures ANCOVA was performed with stimulation type (active vs sham tDCS) and moral domain (altruistic vs deontological) as withinsubject factors and DS-R score as covariate.

Lastly, to investigate whether increased levels of disgust and deontological morality after stimulation of the insular region would be stronger in participants with higher obsessive-compulsive dispositional tendencies, Pearson correlations were performed between scores on the OCI-R, SRAS, MFQ, PIOS-R, and DS-R and $\Delta \mathrm{HRV}_{\text {stim, }} \Delta$ disgust $_{\text {stim }}$, ratio of disgust-related words over total recognized words, and moral judgment to the deontological and altruistic vignettes.

\section{Results}

No adverse effects occurred, with the exception of commonly reported side effects as tingling and itching. Sham and active tDCS blinding guessing was beyond chance.

Table 1 shows sex differences for the main variables of the study. Women were characterized by higher levels of depressive symptoms $(t$ $(35)=2.13, p=.04)$ and disgust sensitivity $(t(35)=2.86, p=.007)$ compared to men. No other sex differences emerged.

Table 2 illustrates the effect of active vs sham tDCS on the elicitation of disgust. When controlling for disgust sensitivity (DS-R scores), during active (compared to sham) tDCS participants reported higher subjective levels of disgust $\left(\mathrm{F}(1,35)=4.99, p=.03 ; \eta_{\mathrm{p}}{ }^{2}=0.12\right)$ and completed more words in terms of cleaning/dirtiness $(\mathrm{F}(1,35)=26.88$, $p<.0001 ; \eta_{\mathrm{p}}{ }^{2}=0.43$ ), suggesting the elicitation of the emotion of
Table 1

Gender differences (means \pm standard errors) in socio-demographic, dispositional, and physiological variables.

\begin{tabular}{llll}
\hline & Women $(\boldsymbol{n}=\mathbf{2 5})$ & Men $(\boldsymbol{n}=\mathbf{1 2})$ & $\mathbf{t} / \boldsymbol{\chi}^{\mathbf{2}}$ \\
\hline Age (years) & $27.2 \pm 1.01$ & $26 \pm 1.47$ & 0.65 \\
Education & $4 \mathrm{C} ; 18 \mathrm{G} ; 3 \mathrm{P}$ & $6 \mathrm{C} ; 5 \mathrm{G} ; 1 \mathrm{P}$ & 4.77 \\
Exercise (h/w) & $1.8 \pm 0.53$ & $1.3 \pm 0.77$ & 0.59 \\
CES-D & $21.5 \pm 1.33$ & $16.5 \pm 1.92$ & $2.13^{*}$ \\
STAI-t & $43.9 \pm 2.04$ & $40.9 \pm 2.95$ & 0.85 \\
DS-R & $55.9 \pm 2.32$ & $44.3 \pm 3.35$ & $2.86^{*}$ \\
PIOS-R & $14.5 \pm 2.55$ & $12.5 \pm 3.53$ & 0.51 \\
SRAS & $29 \pm 2.05$ & $30.5 \pm 2.96$ & 0.42 \\
OCI-R & $25.6 \pm 2.31$ & $19.1 \pm 3.34$ & 1.62 \\
HR (bpm) & $76 \pm 2.11$ & $80.9 \pm 2.78$ & 1.39 \\
HRV (ms) & $34.7 \pm 2.41$ & $35.1 \pm 3.51$ & 0.09
\end{tabular}

Note: C, College; G, Graduate; P, Postgraduate; h/w = hours per week; CES-D = Center for Epidemiologic Studies Depression Scale; STAI- $t=$ State Trait Anxiety Inventory, Trait form; DS-R = Disgust Scale-Revised; PIOS-R = Penn Inventory of Scrupulosity-Revised; SRAS = Self-Report Altruism Scale; OCI-R = Obsessive-Compulsive Inventory-Revised. HR = Heart Rate (Averaged baseline scores); HRV = Heart Rate Variability (Averaged baseline scores); ${ }^{*} p<.05$.

Table 2

Mean scores ( \pm standard errors) for active tDCS stimulation (vs sham) on selfreported $(\Delta=$ post-tDCS momentary affect minus baseline momentary affect), physiological ( $\Delta=$ HRV during tDCS minus HRV at baseline), and implicit measures ( $\Delta=$ ratio of disgust-related words to the total of recognized words).

\begin{tabular}{llll}
\hline & Stim $(\boldsymbol{n}=\mathbf{3 7})$ & Sham $(\boldsymbol{n}=\mathbf{3 7 )}$ & $\boldsymbol{F}(\boldsymbol{d} \boldsymbol{f})$ \\
\hline$\Delta$ Shame & $0.19 \pm 0.12$ & $0.08 \pm 0.08$ & $0.67(1,35)$ \\
$\Delta$ Sad & $0.00 \pm 0.11$ & $-0.16 \pm 0.09$ & $2.70(1,35)$ \\
$\Delta$ Fear & $-0.13 \pm 0.12$ & $-0.35 \pm 0.12$ & $2.11(1,35)$ \\
$\Delta$ Disgust & $0.05 \pm 0.08$ & $-0.08 \pm 0.09$ & $4.99^{*}(1,35)$ \\
$\Delta$ Anger & $0.00 \pm 0.12$ & $-0.19 \pm 0.11$ & $1.50(1,35)$ \\
$\Delta$ Pity & $0.13 \pm 0.11$ & $-0.16 \pm 0.11$ & $5.80^{*}(1,35)$ \\
$\Delta$ Altruistic guilt & $-0.14 \pm 0.11$ & $-0.19 \pm 0.10$ & $0.12(1,35)$ \\
$\Delta$ Deontological guilt & $-0.08 \pm 0.08$ & $0.03 \pm 0.06$ & $2.87(1,35)$ \\
$\Delta$ Happy & $-0.11 \pm 0.09$ & $-0.22 \pm 0.11$ & $0.58(1,35)$ \\
$\Delta$ HR & $-0.03 \pm 0.56$ & $0.73 \pm 0.68$ & $0.62(1,28)$ \\
$\Delta$ HRV & $4.56 \pm 1.39$ & $-0.89 \pm 1.91$ & $4.50^{*}(1,26)$ \\
$\Delta$ Words & $0.38 \pm 0.04$ & $0.17 \pm 0.02$ & $26.88^{\text {*** }}(1,35)$ \\
\hline
\end{tabular}

Note: HR = Heart Rate; HRV = Heart Rate Variability.

$* p<.05$.

$* * p<.0001$.

disgust. Similarly, greater pre- to post-stimulation HRV was observed during the active tDCS condition compared to sham $(\mathrm{F}(1,26)=4.50$, $p=.04 ; \eta_{\mathrm{p}}{ }^{2}=0.15$ ), consistently with the elicitation of disgust at a physiological level. Table 2 also shows a significant effect of active vs sham tDCS on pity $\left(\mathrm{F}(1,35)=5.80, p=.02 ; \eta_{\mathrm{p}}{ }^{2}=0.14\right)$, with increases in self-reported levels of pity after active tDCS.

Analysis on the moral vignettes yielded a main effect of type of vignette (deontological vs altruistic), with altruistic vignettes rated as more morally wrong irrespective of stimulation $(\mathrm{F}(1,36)=4.67$, $\left.p=.04, \eta_{\mathrm{p}}{ }^{2}=0.12\right)$. A marginally significant effect of stimulation type $\mathrm{x}$ vignette type also emerged $\left(\mathrm{F}(1,36)=3.51, p=.06, \eta_{\mathrm{p}}{ }^{2}=0.10\right)$. The covariate (DS-R score) did not play a significant role in the model ( $F$ $\left.(1,35)=.02 ; \mathrm{p}=.88 ; \eta_{\mathrm{p}}^{2}=0.001\right)$.

As depicted in Fig. 1, the type of stimulation only had an effect on deontological vignettes, which were judged as more morally wrong during active $(11.55 \pm 1.21)$ compared to sham stimulation (11.22 \pm 1.37 ), with a small-to-medium effect size (Cohen's $d=0.26$ for post-hoc comparison). No difference emerged for altruistic vignettes $(11.73 \pm 1.54$ vs $11.83 \pm 1.74$ during active and sham tDCS, respectively).

Notably, $\Delta$ disgust $_{\text {stim }}$ was significantly correlated with both $\Delta$ $\mathrm{HRV}_{\text {stim }}(r=0.55 ; p=.001)$ and the word ratio $(r=0.41 ; p=.01)$, somehow supporting the reliability of our implicit, physiological, and 


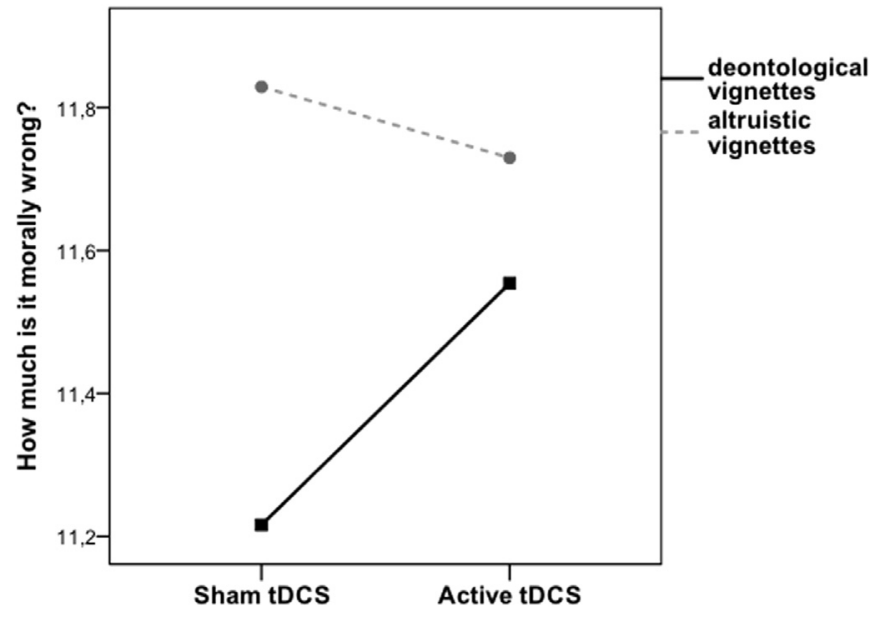

Fig. 1. Effects of active versus sham tDCS on moral judgment in the deontological and in the altruistic domains. Note. The total score for "how much is it morally wrong" in each domain (i.e., altruistic and deontological) represents the sum of the scores obtained on each category belonging to that domain, divided by the number of categories belonging to that domain, i.e. the average (e.g., for the altruistic domain: sum of the scores obtained on animal care, emotional care, and physical human care divided by 3; the total score ranges from 3 to 15 for each domain).

subjective measures of disgust. Moreover, scores on the PIOS were significantly correlated with both $\Delta$ disgust $_{\text {stim }}(r=0.53 ; p=.001)$ and $\Delta \operatorname{HRV}_{\text {stim, }}(r=0.60 ; p=.001)$. A significant association also emerged between scores on the SRAS and moral judgment to the altruistic vignettes only $(r=0.41 ; p=.01)$. On the other hand, moral judgment to the deontological vignettes was associated with scores on the OCI-R $(r=0.33 ; p=.05)$ and particularly with the washing subscale of the OCI-R $(r=0.37 ; p=.03)$.

\section{Discussion}

The broad aim of the present study was to further explore the existence of a unique association between deontological morality and disgust. Capitalizing on preexisting evidence on the association between deontological guilt and activation of the insula (Basile et al., 2011), we hypothesized that non-invasive stimulation of the insular cortex by tDCS would enhance: 1) subjective, implicit, and physiological levels of disgust; 2) moral judgment in the deontological but not in the altruistic domain.

As expected, tDCS stimulation over T3 elicited disgust as indicated by our implicit and physiological measures, and by self-reports. Importantly, these measures were significantly inter-correlated. Such association is not unanticipated, as Mossink et al. (2015) also found an association between implicit measure of affect and cortisol.

The present study also replicated results from previous studies conducted in favor of the notion that electrical stimulation applied over the temporal lobe (T3) can reach surrounding deep cerebral areas like the insula (Lang et al., 2005), with significant effects on parasympathetic modulation of HR (Montenegro et al., 2011; Okano et al., 2015; Petrocchi et al., 2017; Piccirillo et al., 2016). However, none of these previously conducted studies interpreted the increase in HRV that followed tDCS stimulation as due to the elicitation of the emotion of disgust. This is somewhat surprising, given the recognized role of this brain area in disgust sensitivity (Lane et al., 1997; Phillips et al., 1997). Our implicit measure (i.e., the word-fragment completion task), as well as physiological and subjective measures, all point to increased levels of disgust after active tDCS stimulation, suggesting that this specific emotion may indeed be the trigger for the increased HRV, following tDCS stimulation over T3.

Following the suggestion of Landy and Goodwin (2015) we used an induction that does not imply the transgression of a moral norm (i.e., participants voluntarily consented to participate to a tDCS study and the sham condition was identical to the stimulation condition). Results are not conclusive but support a relationship between disgust and morality, particularly in the deontological domain. The present study does not aim to unmask the type of association between these two constructs (amplification or elicitation hypotheses), as it only showed that tDCS over T3 simultaneously enhances disgust and the severity of moral judgments in the deontological domain. In our view, the indirect stimulation of the insular area in healthy participants increases the association between disgust and deontological morality and this models a distinctive feature of OCD (e.g., Basile et al., 2014; Mancini and Gangemi, 2015).

In line with the above assumption, the fact that participants generally rated the vignettes as more morally wrong in the altruistic domain may be due to the fact that the sample was not composed of individuals with OCD, who would be more sensitive to deontological morality (e.g., Basile et al., 2014; Mancini and Gangemi, 2015). Indeed, a positive correlation emerged between scores on the OCI-R and moral judgment in the deontological domain. On the other hand, scores on the SRAS were associated with moral judgment only in the altruistic domain, supporting the existence of two forms of morality and the method used to categorize the vignettes.

The interaction between stimulation type and vignette type was only marginally significant $(p=.06)$, however, the effects were in the expected direction, suggesting that the active stimulation of the insula enhanced morality only in the deontological domain. Cannon et al. (2011) also supported this notion using facial muscle activity, showing that facial disgust was highest in response to purity violations; furthermore, subsequent moral judgments about purity and fairness (but not harm violations) were predicted by facial disgust.

The role of the temporal cortex, and particularly the insular region, in moral judgment has been recognized in a review on the functional and clinical neuroanatomy of morality (Fumagalli and Priori, 2012). The present study is not the first to highlight a specific involvement of the insula in moral processing and norm violations implicated in deontological judgment (see Huebner et al., 2009). Of note are the scores on the VAS, for which we found only a weak effect of active tDCS on disgust and also on pity. Moreover, we did not find the expected effect of active tDCS on deontological guilt, which would have been in line with our hypothesis. Whereas disgust is a primary emotion, pity and deontological guilt are secondary emotions. As such, they are more strongly affected by the way in which individuals interpret the specific situation that they are experiencing. In the present study, we have reassessed participants' momentary affect at the end of the protocol, i.e. after the moral task. It is likely that reading a series of moral scenarios influences participants' interpretation of their current emotional state. Further studies need to evaluate how tDCS stimulation over T3 specifically and differentially affects primary and secondary emotions.

A major limitation of the current study is that topographical specificity of the tDCS effect has not been demonstrated (i.e., by stimulating a control site of the brain), thus allowing for the possibility that the observed findings are non-specific. Second, it would have been of major interest to also include anodal tDCS of the right temporal cortex, given that also the right insula forms a core of the central autonomic network (Beissner et al., 2013 for a meta-analysis). Third, although we have used a standardized set of the vignettes and we did our best to adapt them to the Italian culture by running a preliminary study, our categorization of the moral foundations in altruistic and deontological morality might be questionable. Lastly, despite our effort to recruit among the general population via the use of social networks and advertisements, the majority of the sample consisted of graduate and postgraduate students, which may hinder the generalization of the results.

In the case of successful replication, the present study has important clinical implications, considering that both deontological morality and 
disgust play a central role in OCD (Mancini, 2016; Power and Dalgleish, 2008). Here, we have used tDCS to increase cortical excitability in the insular region, but it is known that cathodal tDCS has the potential to decrease excitability (Nitsche and Paulus, 2000), although the canonical assumption "anodal excitation, cathodal inhibition" has not always been replicated" (AeCi-effect; Jacobson et al., 2012). Thus, there is the need to replicate and extend present findings in non-pathological individuals with the inclusion of a cathodal condition in the experimental design. In the case of successful replication, the next step would be to run a randomized controlled trial on OCD patients to test whether these effects are applicable to the clinical setting. It is important to note that Basile et al. (2014) found that -when faced with stimuli evoking deontological guilt- patients with OCD showed decreased activation in the insula compared to controls. The authors interpret these data as suggesting that this pattern may reflect patients' cerebral efficiency, due to the frequent exposure to deontological feelings (neural efficiency hypothesis; Del Percio et al., 2009). Thus, further investigation of the brain correlates of deontological morality in OCD is needed before noninvasive brain stimulation techniques can be used as therapeutic means.

\section{Acknowledgments}

The study has been funded by the Italian Ministry of Health Young Researcher Grant (GR-2011-02348232). The authors would like to thank Tania Moretta for her participation during the planning phase of the study and Dr. Frances Meeten for editing and proofreading the manuscript.

\section{Financial disclosures}

The authors report no biomedical financial interests or potential conflicts of interest.

\section{References}

Anderson, C.A., Carnagey, N.L., Eubanks, J., 2003. Exposure to violent media: the effects of songs with violent lyrics on aggressive thoughts and feelings. J. Personal. Soc. Psychol. 84, 960-971.

Babic, T., Browning, K.N., 2014. The role of vagal neurocircuits in the regulation of nausea and vomiting. Eur. J. Pharmacol. 722, 38-47.

Basile, B., Mancini, F., Macaluso, E., Caltagirone, C., Bozzali, M., 2014. Abnormal processing of deontological guilt in obsessive-compulsive disorder. Brain Struct. Funct. 219, 1321-1331.

Basile, B., Mancini, F., Macaluso, E., Caltagirone, C., Frackowiack, R., Bozzali, M., 2011 Deontological and altruistic guilt: evidence for distinct neurobiological substrates. Hum. Brain Mapp. 32, 229-232.

Beissner, F., Meissner, K., Bär, K.J., Napadow, V., 2013. The autonomic brain: an activation likelihood estimation meta-analysis for central processing of autonomic function. J. Neurosci. 33, 10503-10511.

Cannon, P.R., Schnall, S., White, M., 2011. Transgressions and expressions: affective facial muscle activity predicts moral judgments. Soc. Psychol. Personal. Sci. 2, 325-331.

Caseras, X., Mataix-Cols, D., An, S.K., Lawrence, N.S., Speckens, A., Giampietro, V., Brammer, M.J., Phillips, M.L., 2007. Sex differences in neural responses to disgusting visual stimuli: implications for disgust-related psychiatric disorders. Biol. Psychiatry 62, 464-471.

Cisler, J.M., Olatunji, B.O., Lohr, J.M., 2009. Disgust fear and the anxiety disorders: a critical review. Clin. Psychol. Rev. 29, 34-46.

Clifford, S., Iyengar, V., Cabeza, R., Sinnott-Armstrong, W., 2015. Moral foundations vignettes: a standardized stimulus database of scenarios based on moral foundations theory. Behav. Res. Methods 47, 1178-1198.

Davey, C.G.L., 2011. Disgust: the disease-avoidance emotion and its dysfunctions. Philos. Trans. R. Soc. Lond. B Biol. Sci. 366, 3453-3465.

D’Olimpio, F., Mancini, F., 2014. Role of deontological guilt in obsessive-compulsive disorder-like checking and washing behaviors. Clin. Psychol. Sci. 2, 727-739.

Del Percio, C., Babiloni, C., Marzano, N., Iacoboni, M., Infarinato, F., Vecchio, F., Lizio, R., Aschieri, P., Fiore, A., Toràn, G., Gallamini, M., Baratto, M., Eusebi, F., 2009. Neural efficiency" of athletes' brain for upright standing: a high-resolution EEG study. Brain Res. Bull. 79, 193-200.

Denny, E.B., Hunt, R.R., 1992. Affective valence and memory in depression: dissociation of recall and fragment completion. J. Abnorm. Psychol. 101, 575-580.

Earp, B.D., Everett, J.A.C., Madva, E.N., Hamlin, J.K., 2014. Out, damned spot: can the "Macbeth Effect" be replicated? Basic Appl. Soc. Psychol. 36, 91-98.

Ekman, P., Levenson, R.W., Friesen, W.V., 1983. Autonomic nervous system activity distinguishes among emotions. Science 221, 1208-1210.
Fava, G.A., 1983. Assessing depressive symptoms across cultures: Italian validation of the CES-D self-rating scale. J. Clin. Psychol. 39, 249-251.

Foa, E.B., Huppert, J.D., Leiberg, S., Langner, R., Kichic, R., Hajcak, G., Salkovskis, P.M., 2002. The obsessive-compulsive Inventory: development and validation of a short version. Psychol. Assess. 14, 485-496.

Fumagalli, M., Ferrucci, R., Mameli, F., Marceglia, S., Mrakic-Sposta, S., Zago, S., Lucchiari, C., Consonni, D., Nordio, F., Pravettoni, G., Cappa, S., Priori, A., 2010. Gender-related differences in moral judgments. Cogn. Process. 11, 219-226.

Fumagalli, M., Priori, A., 2012. Functional and clinical neuroanatomy of morality. Brain 135, 2006-2021.

Gandiga, P.C., Hummel, F.C., Cohen, L.G., 2006. Transcranial DC stimulation (tDCS): a tool for double-blind sham-controlled clinical studies in brain stimulation. Clin. Neurophysiol. 117, 845-850.

Graham, J., Nosek, B.A., Haidt, J., Iyer, R., Koleva, S., Ditto, P.H., 2011. Mapping the Moral Domain. J. Personal. Soc. Psychol. 101, 366-385.

Haidt, J., 2013. Moral psychology for the twenty-first century. J. Moral Educ. 42, 281-297.

Herba, J.K., Rachman, S., 2007. Vulnerability to mental contamination. Behav. Res. Ther. 45, 2804-2812.

Huebner, B., Dwyer, S., Hauser, M., 2009. The role of emotion in moral psychology. Trends Cogn. Sci. 13, 1-6.

Iyer, R., Koleva, S., Graham, J., Ditto, P., Haidt, J., 2012. Understanding libertarian morality: the psychological dispositions of self-identified libertarians. PLoS One 7, e42366.

Jacobson, L., Koslowsky, M., Lavidor, M., 2012. tDCS polarity effects in motor and cognitive domains: a meta-analytical review. Exp. Brain Res. 216, 1-10.

Jennings, J.R., Kamarck, T., Stewart, C., Eddy, M., Johnson, P., 1992. Alternate cardiovascular baseline assessment techniques: vanilla or resting baseline. Psychophysiology 29, 742-750.

Johnson, R.E., Steinman, L., 2009. The use of implicit measures for organizational research: an empirical example. Can. J. Behav. Sci. 41, 202-212.

Kang, L.L., Rowatt, W.C., Fergus, T.A., 2016. Moral foundations and obsessive-compulsive symptoms: a preliminary examination. J. Obsessive Compuls. Relat. Disord. 11, 22-30.

Koenig, J., Thayer, J.F., 2016. Sex differences in healthy human heart rate variability: a meta-analysis. Neurosci. Biobehav. Rev. 64, 288-310.

Landy, J.F., Goodwin, G.P., 2015. Does incidental disgust amplify moral judgment? A meta-analytic review of experimental evidence. Perspect. Psychol. Sci. 10, 518-536.

Lane, R.D., Reiman, E.M., Ahern, G.L., Schwartz, G.E., Davidson, R.J., 1997. Neuroanatomical correlates of happiness, sadness, and disgust. Am. J. Psychiatry 154, 929-933.

Lang, N., Siebner, H.R., Ward, N.S., Lee, L., Nitsche, M.A., Paulus, W., Rothwell, J.C., Lemon, R.N., Frackowiak, R.S., 2005. How does transcranial DC stimulation of the primary motor cortex alter regional neuronal activity in the human brain? Eur. J. Neurosci. 22, 495-504.

Lee, S.W.S., Schwarz, N., 2011. Wiping the slate clean: psychological consequences of physical cleansing. Curr. Dir. Psychol. Sci. 20, 307-311.

Mancini, F., 2016. La mente ossessiva. Raffaello Cortina, Milano.

Mancini, F., Gangemi, A., 2015. Deontological guilt and obsessive compulsive disorder. J. Behav. Ther. Exp. Psychiatry 49, 157-163.

Mancini, F., Gangemi, A., 2017. Obsessive patients and their guilt. Psychopathol. Rev. 7, 155-168.

Mataix-Cols, D., Rosario-Campos, M.C., Leckman, J.F., 2005. A multidimensional model of obsessive-compulsive disorder. Am. J. Psychiatry 162, 228-238.

Montenegro, R.A., Farinatti Pde, T., Fontes, E.B., Soares, P.P., Cunha, F.A., Gurgel, J.L., Porto, F., Cyrino, E.S., Okano, A.H., 2011. Transcranial direct current stimulation influences the cardiac autonomic nervous control. Neurosci. Lett. 497, 32-36.

Monte-Silva, K., Kuo, M.F., Liebetanz, D., Paulus, W., Nitsche, M.A., 2010. Shaping the optimal repetition interval for cathodal transcranial Direct Current Stimulation (tDCS). J. Neurophysiol. 103, 1735-1740.

Mossink, J.C.L., Verkuil, B., Burger, A.M., Tollenaar, M.S., Brosschot, J.F., 2015. Ambulatory assessed implicit affect is associated with salivary cortisol. Front. Psychol. 6, 111.

Nitsche, M.A., Paulus, W., 2000. Excitability changes induced in the human motor cortex by weak transcranial direct current stimulation. J. Physiol. 527, 633-639.

Okano, A.H., Fontes, E.B., Montenegro, R.A., Farinatti, Pde, T., Cyrino, E.S., Li, L.M., Bikson, M., Noakes, T.D., 2015. Brain stimulation modulates the autonomic nervous system, rating of perceived exertion and performance during maximal exercise. Br. J. Sports Med. 18, 1213-1218.

Olatunji, B.O., Williams, N.L., Tolin, D.F., Abramowitz, J.S., Sawchuk, C.N., Lohr, J.M., Elwood, L.S., 2007a. The disgust scale: item analysis, factor structure, and suggestions for refinement. Psychol. Assess. 19, 281-297.

Olatunji, B.O., Abramowitz, J.S., Williams, N.L., Connolly, K.M., Lohr, J.M., 2007b. Scrupulosity and obsessive-compulsive symptoms: confirmatory factor analysis and validity of the Penn Inventory of Scrupulosity. J. Anx. Dis. 21, 771-787.

Ottaviani, C., Collazzoni, A., D’Olimpio, F., Moretta, T., Mancini, F., 2018. I obsessively clean because my deontological guilt makes me feel physiologically disgusted!. J. Obsessive Compuls. Relat. Disord. Adv. Online Publ.

Ottaviani, C., Mancini, F., Petrocchi, N., Medea, B., Couyoumdjian, A., 2013. Autonomic correlates of physical and moral disgust. Int. J. Psychophysiol. 89, 57-62.

Park, G., Vasey, M.W., Van Bavel, J.J., Thayer, J.F., 2014. When tonic cardiac vagal tone predicts changes in phasic vagal tone: the role of fear and perceptual load. Psychophysiology 51, 419-426.

Pedrabissi, L., Santinello, M., 1989. Nuova versione italiana dello STAI forma Y [New Italian version of the STAI form Y]. Organizzazioni Speciali, Firenze, Italy.

Petrocchi, N., Piccirillo, G., Fiorucci, C., Moscucci, F., Di Iorio, C., Mastropietri, F., 
Parrotta, I., Pascucci, M., Magrì, D., Ottaviani, C., 2017. Transcranial direct current stimulation enhances soothing positive affect and vagal tone. Neuropsychologia 96, 256-261.

Phillips, M.L., Young, A.W., Senior, C., Brammer, M., Andrew, C., Calder, A.J., Bullmore, E.T., Perrett, D.I., Rowland, D., Williams, S.C., Gray, J.A., David, A.S., 1997. A specific neural substrate for perceiving facial expressions of disgust. Nature 389 , 495-498.

Piccirillo, G., Ottaviani, C., Fiorucci, C., Petrocchi, N., Moscucci, F., Di Iorio, C., Mastropietri, F., Parrotta, I., Pascucci, M., Magrì, D., 2016. Transcranial direct current stimulation improves the QT variability index and autonomic cardiac control in healthy subjects over sixty years old. Clin. Interv. Aging 11, 1687-1695.

Power, M., Dalgleish, T., 2008. Cognition and Emotion: From Order to Disorder, 2nd ed. Taylor Francis, Hove, UK.

Pujol, J., Blanco-Hinojo, L., Coronas, R., Esteba-Castillo, S., Rigla, M., Martínez-Vilavella, G., Deus, J., Novell, R., Caixàs, A., 2018. Mapping the sequence of brain events in response to disgusting food. Hum. Brain Mapp. 39, 369-380.

Rachman, S., 2004. Fear of contamination. Behav. Res. Ther. 42, 1227-1255.

Rachman, S., Radomsky, A.S., Elliott, C.M., Zysk, E., 2012. Mental contamination: the perpetrator effect. J. Behav. Ther. Exp. Psychiatry 43, 587-593.

Radloff, L.S., 1977. The CES-D scale: a self-report depression scale for research in the general population. Appl. Psychol. Meas. 1, 385-401.

Rauch, S.L., Dougherty, D.D., Shin, L.M., Alpert, N.M., Manzo, P., Leahy, L., et al., 1998. Neural correlates of factor-analyzed OCD symptom dimensions: a PET study. CNS Spectr. 3, 37-43.

Reuven, O., Liberman, N., Dar, R., 2014. The effect of physical cleaning on threatened morality in individuals with obsessive-compulsive disorder. Clin. Psychol. Sci. 2 , 224-229.

Rohrmann, S., Hopp, H., 2008. Cardiovascular indicators of disgust. Int. J. Psychophysiol. 68, 201-208.
Rozin, P., Lowery, L., Imada, S., Haidt, J., 1999. The CAD triad hypothesis: a mapping between three moral emotions (contempt, anger, disgust) and three moral codes (community, autonomy, divinity). J. Personal. Soc. Psychol. 76, 574-586.

Rushton, J.P., Chrisjohn, R.D., Fekken, G.C., 1981. The altruistic personality and the selfreport altruism scale. Personal. Individ. Dif. 2, 293-302.

Schimel, J., Greenberg, J., Martens, A., 2003. Evidence that projection of a feared trait can serve a defensive function. Personal. Soc. Psychol. Bull. 29, 969-979.

Schlegel, T.T., Brown, T.E., Wood, S.J., Benavides, E.W., Bondar, R.L., Stein, F., Moradshahi, P., Harm, D.L., Fritsch-Yelle, J.M., Low, P.A., 2001. Orthostatic intolerance and motion sickness after parabolic flight. J. Appl. Physiol. 90, 67-82.

Schnall, S., Benton, J., Harvey, S., 2008a. With a clean conscience: cleanliness reduces the severity of moral judgments. Psychol. Sci. 19, 1219-1222.

Schnall, S., Haidt, J., Clore, G.L., Jordan, A., 2008b. Disgust as embodied moral judgment Personal. Soc. Psychol. Bull. 34, 1096-1109.

Spielberger, C.D., Gorsuch, R.L., Lushene, R.E., 1970. STAI Manual. Consulting Psychologists Press, Palo Alto, CA.

Tarvainen, M.P., Niskanen, J.P., Lipponen, J.A., Ranta-Aho, P.O., Karjalainen, P.A., 2014. Kubios HRV-heart rate variability analysis software. Comput. Methods Prog. Biomed. $113,210-220$.

Task Force of the European Society of Cardiology, 1996. Heart rate variability standards of measurement, physiological interpretation, and clinical use. Eur. Heart J. 17, 354-381.

Tobia, K.P., 2015. The effects of cleanliness and disgust on moral judgment. Philos. Psychol. 28, 556-568.

Winkielman, P., Berridge, K.C., 2004. Unconscious emotion. Am. Psychol. Soc. 13, 120-123.

Zhong, C.B., Liljenquist, K., 2006. Washing away your sins: threatened morality and physical cleansing. Science 313, 1451-1452. 